BMJ Open

Diabetes

Research

\& Care

\title{
Lost opportunities to prevent early onset type 2 diabetes mellitus after a pregnancy complicated by gestational diabetes
}

\author{
Judith A Bernstein, ${ }^{1,2}$ Lois McCloskey, ${ }^{3}$ Christina M Gebel, ${ }^{3}$ Ronald E Iverson, ${ }^{4}$ \\ Aviva Lee-Parritz ${ }^{4}$
}

To cite: Bernstein JA, McCloskey L, Gebel CM, et al. Lost opportunities to prevent early onset type 2 diabetes mellitus after a pregnancy complicated by gestational diabetes. BMJ Open Diabetes Research and Care 2016;4:e000250. doi:10.1136/bmjdrc-2016000250

Received 18 April 2016 Revised 13 May 2016 Accepted 26 May 2016

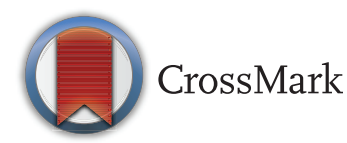

\footnotetext{
${ }^{1}$ Department of Community Health Sciences, Boston University School of Public Health, Boston,

Massachusetts, USA

${ }^{2}$ Department of Emergency Medicine, Boston University School of Medicine, Boston, Massachusetts, USA ${ }^{3}$ Boston University School of Public Health, Boston, Massachusetts, USA

${ }^{4}$ Boston University School of Medicine, Boston,

Massachusetts, USA
}

Correspondence to Dr Judith A Bernstein; jbernste@bu.edu

\section{ABSTRACT}

Objectives: Gestational diabetes mellitus (GDM) greatly increases the risk of developing diabetes in the decade after delivery, but few women receive appropriately timed postpartum glucose testing (PPGT) or a referral to primary care $(P C)$ for continued monitoring. This qualitative study was designed to identify barriers and facilitators to testing and referral from patient and providers' perspectives.

Methods: We interviewed patients and clinicians in depth about knowledge, values, priorities, challenges, and recommendations for increasing PPGT rates and PC linkage. Interviews were coded with NVIVO data analysis software, and analyzed using an implementation science framework.

Results: Women reported motivation to address GDM for the health of the fetus. Most women did not anticipate future diabetes for themselves, and focused on delivery outcomes rather than future health risks. Patients sought and received reassurance from clinicians, and were unlikely to discuss early onset following GDM or preventive measures. PPGT barriers described by patients included provider not mentioning the test or setting it up, transportation difficulties, work responsibilities, fatigue, concerns about fasting while breastfeeding, and timing of the test after discharge from obstetrics, and no referral to PC for follow-up. Practitioners described limited communication among multiple care providers during pregnancy and delivery, systems issues, and separation of obstetrics from PC.

Conclusions: Patients' barriers to PPGT included low motivation for self-care, structural obstacles, and competing priorities. Providers reported the need to balance risk with reassurance, and identified systems failures related to test timing, limitations of electronic medical record systems (EMR), lack of referrals to PC, and inadequate communication between specialties. Prevention of early onset has great potential for medical cost savings and improvements in quality of life.

\section{INTRODUCTION}

Onset of type 2 diabetes mellitus (T2DM) after a pregnancy complicated by gestational diabetes mellitus (GDM) is highly preventable

\section{Key messages}

- The risk for early onset of type 2 diabetes mellitus in the decade following gestational diabetes mellitus (GDM) is high and preventable, but most women do not receive postpartum glucose testing or an effective referral to primary care for continued monitoring after GDM.

- Neither clinicians nor patients appear to appreciate the impact of failure to follow-up on health across the life cycle and the emergence of chronic disease in later life.

- Timing of the postpartum glucose test in the gap between specialties contributes to fragmentation of care.

and a major contributor to the increasing prevalence of T2DM, a significant cause of chronic illness and disability among older women. It is estimated that $30 \%$ of the women with T2DM in the USA in $2010^{12}$ were originally diagnosed with $\mathrm{GDM}^{3}{ }^{3}{ }^{4}$ which increases the risk of T2DM by sevenfold. ${ }^{5}$ By 10 years postpartum, the cumulative incidence of T2DM after a GDM pregnancy is $60 \%$, and each re-occurring GDM pregnancy contributes to the risk of T2DM onset. $^{6-9}$

Despite a strong body of evidence supporting the effectiveness and cost-effectiveness of preventive monitoring and early detection of T2DM,${ }^{10-20}$ fewer than half of women with GDM receive glucose testing as recommended by professional guidelines within 84 days of delivery and every $1-3$ years thereafter, ${ }^{4} 2122$ with especially low rates in the highest risk groups, such as minority women and those with the most severe GDM. ${ }^{23} 24$ GDM presents women and their providers with an impressive opportunity for prevention of a major cause of chronic illness and disability among mid-life and older women. ${ }^{25}$

We conducted a qualitative study with women and their providers, interviewing 
women in the third trimester after a GDM diagnosis and again postpartum to identify barriers and facilitators to postpartum glucose testing (PPGT) and referral to primary care (PC) for continued monitoring. We then used the Consolidated Framework for Implementation Research (CFIR) ${ }^{26}$ to assess contributions to the likelihood of testing and linkage from four domains: intervention attributes (postpartum testing and transition to PC postdelivery); the characteristics of individuals (defined as women's values, beliefs, resources, and life circumstances); the inner context (physician characteristics, values, knowledge and beliefs, and system issues); and the outer context (policies and resources).

\section{RESEARCH DESIGN AND METHODS}

\section{Study design and sample}

In this descriptive, qualitative study, conducted during 2012-2013, we used convenience sampling to recruit and interview clinicians whose practices include the management of GDM during and/or after pregnancy and their patients in an urban safety net hospital. A 30-min interview occurred in practitioners' offices or by telephone. Patients enrolled during the third trimester after receiving a GDM diagnosis; a 1-hour interview with patients occurred at 10-14 weeks postdelivery in their homes. This study (H-29330) was approved by the Boston University Institutional Review Board in an expedited review process in accordance with 45 CFR 46.110 and 21 CFR 56.110.

\section{Data collection}

We used a semi-structured interview format to elicit patients' and clinicians' experiences and perspectives on postpartum testing and linkage to PC after GDM. Our questions to patients and clinicians included knowledge, values, and priorities; sources of information, challenges, and recommendations for a single change that could be instituted to increase rates of testing and linkage. All interviews were conducted by the principal investigator, project manager, or research assistant in English, Spanish, or Haitian Creole, either in person or by telephone, and then audiotaped and transcribed verbatim.

\section{Data analysis}

Our analysis followed standard procedures and methods of qualitative analysis. ${ }^{27}$ We used the first five transcripts to develop a coding scheme and test for inter-rater reliability ( $\kappa$ coefficient $>0.70$ ). We then used cloud-based qualitative data analysis software (Dedoose) to apply this coding scheme to the text and group material by themes.

We present findings for the four domains of the CFIR model: intervention, individual characteristics, inner context, and outer context. For each domain, we use illustrative quotes, presented in table 1 , to flesh out the meaning of themes that emerged from patient and provider interviews, identifying the source of the quote by practitioner category or patient characteristics.

\section{RESULTS}

Interviews were conducted with 25 clinicians ( 7 obstetricians, 5 family medicine physicians, 8 certified nurse midwives, 2 endocrinologists, and 3 internal medicine physicians) and with 27 patients (13 in English, 7 in Spanish, and 7 in Haitian Creole). Key themes from these stakeholder interviews are listed in table 1.

\section{The domain of patient characteristics}

Women described being highly motivated to address their GDM diagnosis for the sake of the child they envisioned from this pregnancy, but less so for themselves. Most were aware of possible consequences of GDM for the child and the seriousness of a diagnosis of diabetes if they actually had diabetes, but most were certain that there would be no future problems after delivery, in part because physicians were reassuring about their ability to provide good care. While some women wanted the truth about their diagnosis and future risks from their physicians ('please don't sugar coat'), some were afraid to hear what was said and thought pregnancy was 'not a time to hear more problems'.

Providers across specialties had major concerns about patients' ability to understand and tolerate risk. On the one hand, interviewees expressed a lot of empathy for the stressors that women who use an urban academic clinic experience in simply trying to feed and house their families. Clinicians recognized the stress that accompanies even an uncomplicated pregnancy, and they wanted to remain positive, to validate the women for attempting to reorder their priorities. As a result, they often opted for a short-term vision of 'Let's work together to make sure you have a healthy baby', and did not go into the possible consequences for the women themselves in the future in depth. In the end, many women leave obstetrical care without understanding that they are at risk for early onset of T2DM, and that they can do something about that risk. Hence, opportunities for prevention are lost.

\section{The domain of intervention attributes}

The postpartum test is problematic for patients because the effort that is involved (showing up early in the morning, at a scheduled time, fasting) is not congruent with having a new baby in the house. Given that contradiction, most women opt to plan activities around the needs of the newborn, not around the needs of the medical care system. Many patients did understand the significance of the postpartum test and referral for continued monitoring after the postpartum period. But for others, drinking sugar to test sugar for the postpartum glucose test did not make sense, and neither did fasting or subjecting yourself to multiple needles. Patients described many challenges to keeping 
Table 1 Key themes from interviewee responses (language spoken by patients (SP and $\mathrm{HC}$ ) is indicated in brackets if not English; practitioner type is indicated in brackets)

\begin{tabular}{ll}
\hline Domain & Category \\
\hline $\begin{array}{l}\text { Patients: what they think } \\
\text { and know }\end{array}$ & Motivation to comply \\
& $\begin{array}{l}\text { Knowledge: consequences } \\
\text { cause concern }\end{array}$
\end{tabular}
cause concern

Knowledge: not worried reconsequences

\section{Competing priorities}

Consequences

Risk versus reassurance

Patient characteristics

Intervention: patients

Intervention: providers sense no sense testing

\section{Comments}

'I take care of what I have to when I have to as far as my baby, and her being inside of me and me being sick taking care of me is taking care of her. So during my pregnancy I was at the doctor's when I was supposed to be on time and it was like at least three times a week I had to be in that hospital. I hated it but I did it for her'.

'Where I come from diabetes are not something like you just play with. We hear that people get amputated from diabetes [or a sore that] doesn't heal because you have sugar and it keeps getting bigger and bigger. I thought that maybe I was at risk and the pregnancy was at risk or maybe the baby, too, might get diabetes'. [SP] 'It's a very treacherous disease'. 'Just, no, you can't take it lightly'. 'Cause you can't be selfish and think of yourself, you have to think about that little person that's growing inside you that's you know, relying on you to do what you need to do in order for them to survive'.

'I had heard people say that there were people who suffer from that, but at the same time, they told me not to worry about it, that's normal this happens to a lot of women but once the girl is born, it's going to be over. It goes away'. [SP] 'I have no diabetes anymore that my blood's all right I'm okay'. [SP]

'They're limited, no money, it's not a priority for them. GDM is not painful, so then they go and talk to their friends and family members who have advice that is logical to them but not medically appropriate'. 'I don't mean to degrade my patients, and I feel like whatever they're doing is the best they can do'. [CNM]

'[They're] concerned for future pregnancies. I don't know if the motivation is find out if I have diabetes'. [CNM]

'You do so well with all the other things, yes it's complex, but you are going to be able to do it really well and we will just take it step by step'. [OB] 'We want to optimize your health there's something there that's a red flag but it's not too bad'. 'You know it's good to stay motivational as opposed to just scare people with diagnoses because that can be not helpful at all'.

Testing and monitoring make

Testing is unpleasant, makes

Challenges to showing up for

Since I wanted to see if I was well because if I had has ended up with that, I would have to continue treatment and to come to terms and I would have to ask for counseling or something to help me cope with that, because I think it would have been very hard for me to know that I ended up with that. At a later time on another appointment they would be monitoring me. My mom died of diabetes, you know, I would have genetic predisposition to that. Every time that I have an appointment they would be checking me. [SP]

'I said that they had given me something sweet that made me throw up. Me, I had a problem with them taking blood. I don't like needles. They take a lot of blood and they don't give me anything to replace it back. That's my problem'. [HC] 'It was funny. They give you the sugar water. How can you give somebody sugar to drink and then you're going to have to test it? They're definitely going to find the sugar'. Oh!, the difficulty right now is because of the winter. He has to work he has to take me. So, it's very difficult to get around'. [SP] 'They wanted to help me [and]they were worried about my baby but they always gave me a schedule that I couldn't do. I couldn't leave work because they could take it away and I knew the situation I was in, I needed to work'. [SP] 'I supposed to ask them to test but I didn't ask because I have no time. Yeah, so now I didn't have time to shower, how I get a blood test?'.

'Most providers know to do it. Whether or not most providers are doing it is a different story'. [Laborist] 'One of the complications is, my understanding is, that we usually do [the test] at 12 weeks. So, we fall into a gap of the shift to primary care. So I didn't recommend it happening. It's not a really easy test to 


\begin{tabular}{lll}
\hline Domain & Category & Comments
\end{tabular}

Inner context

Outer context
The OB collaborative model of care

Links between specialties and sense of expertise

EMR capacity coordinate because we tend to see people up to 6 weeks and then the disengagement happens and it's not ideal. Sometimes l've brought people back for a 12 week visit but it takes that extra step of coordination and for example, about $60 \%$ of patients come for the postpartum visit. So it's a visit that's traditionally missed, the mothers' busy, she has a new baby and if she feels well she does not want to come, so making that link'. [CNM] 'I think most people who come back for their postpartum visit will come back for the test, but if you don't do it that same day then you're asking for an additional visit which ends up just having all of the barriers you have in usual care [and]often times they end up not being able to wait the 2 hours and they say, 'I'm going to have to come back another day. But then some percentage of them don't'. [OB]

'I try to remind the residents to put it in the discharge summaries because not everybody comes back to me as a provider'. [102] 'There are people who fall through the cracks because they don't come to their postpartum visit and it's a question of do we track those patients and follow them and follow and make sure that those things are happening. And honestly right now, that isn't something that has been made a priority'. [CNM] 'Unless they have some complication, I don't really follow them'. [Laborist]

'I think as we go ahead, one of the things that we are all going to be looking at is how do we bridge those specialty care to primary care gaps? How do we bridge that long-term heath vision and how to we make sure the hand offs are really safe?'. [CNM] 'But I don't feel equipped to handle a positive test, so I think that's why I don't have them follow up with me, because I don't know all of the things about the Like, I want, I want them to follow up with their primary care provider, so they can say "Okay, this is how we're going to go forward in the future. And I feel like l'm not equipped to give them that information, so it wheels down to be like 'Test is normal, you know, Bye"'. [CNM] 'I'll be in touch with them whatever the results are, just to make sure that they've got a primary care connection, which can be challenging because sometimes they don't have a primary care provider'. [CNM] 'I feel like we get a lot of things across our desktop and I don't flag normal results to people, you know, generally I don't'. [OB/GYN] 'So in the ideal world they'll come in and they'll get the result and then I could talk to them about that I think that it would be much better to have the results in your hand (sat the last $O B$ visit), already have made the referral, had the doctors name, write it down for them.'. [Laborist]

If it's not in the problem list, the resident who is seeing the patient that day may not know about GDM because he would have to look back at her other laboratories. You see the patient and talk about the baby but not beyond'.

'How do we bridge that long-term health vision and make sure handoffs are really safe?'. [CNM]

'Every woman would get testing in the home and have data funneled into a registry and every woman would get contacted with results and what to do about it.'. [Endocrinologist]

CNM, certified nurse midwife; FM, family medicine physician; HC, Haitian Creole; MFM, maternal fetal medicine; OB, obstetrician; SP, Spanish.

appointments, but highest on the list were transportation, work responsibilities, conflicts with breast feeding, and limited time.

Providers described marked variations in the timing of the postpartum test, the type of test administered, preparation of patients for the test, and scheduling appointments. There was much uncertainty reported about how to make sure the patient has an appointment for testing and how results are known and communicated to the patient. The biologically indicated timing of the test for 8-12 weeks postpartum, when blood sugar levels have dropped if they are going to do so, was 
reported as inherently challenging to implement, since patients are discharged from obstetrical care at 68 weeks. Obstetrical providers described the dilemma of ordering the test, but by the time results come back, the patient is discharged from obstetrical care, and there are no scheduled follow-up appointments. Most providers understood the challenges, but had no way to address them. The need to schedule the appointment for a glucose test for a future date, because of the interval required for glucose to return to normal if it is going to, was reported as a significant obstacle.

\section{The domain of inner context}

Among the patients we interviewed, obstetrical care was delivered through a collaborative model. The clinician who managed their prenatal care was often a different person from the team present in the delivery room and the practitioner the patient saw postdelivery. Providers recognized that communication of a GDM diagnosis and any testing that occurred prior to a postdelivery PC visit fell into the chasm between specialties. Moreover, obstetrical providers recognized the need for follow-up after GDM, but did not feel equipped to 'handle a positive test' or prepared to offer postdelivery monitoring themselves.

Often the only feasible method of communication reported, across professional silos, was the medical record. While EMR holds promise, the providers we interviewed expressed frustration that technology does not seem to have evolved enough to have efficient referral and tracking systems within each specialty, let alone across the gap between obstetrics and PC. One attending physician noted, 'Depending on how many patients I have, sometimes, I have time to read the charts'. A number of providers described telling patients to follow-up with their replace PCP here with primary care provider (PCP) or refer them to a PCP if they did not already have one. Some providers described sending PCPs a flag alerting them to the patient's GDM history and/or postpartum test results. However, some providers reported only flagging charts with positive test results.

\section{The domain of outer context}

Providers and patients talked about the challenges that can accompany referral to $\mathrm{PC}$ if it means finding a provider outside the birthing institution. 'We make sure they see a pediatrician', one CNM said, 'but [primary care] it's not on our radar'. Many patients appeared bewildered by the task of finding a PC provider who would accept their insurance. Several practitioners mentioned that if the patient had a general practitioner's name listed in the record prior to pregnancy, they just put that name in the referral box and told the patient to make an appointment, but did not follow-up to find out if patients were able to resolve barriers to continuing care. A family medicine physician summed up the lack of continuity this way: 'We as a health system are moving toward a patient-centered medical home and a prevention of chronic disease model, but what I'm finding is that because they are pregnant they [pregnant women] kind of get relegated to this whole other category'.

\section{Providers' recommendations for improving rates of postpartum testing and transition to PC}

Interviewees were asked, 'If you could think of a single innovation to increase testing rates and the chance of transition to primary care, what would it be?'. Practitioners suggested several innovations that could be implemented to increase testing rates and improve the chance of successful transition of women diagnosed with GDM to PC. Several interviewees stressed the need to change 'meaningful use' discharge instructions to include an appointment for glucose testing at 12 weeks and the name of a PC source. It was also suggested that the most effective way to foster compliance with PPGT would be to move the test from the laboratory to home, with a visiting nurse administering the 2-hour glucose tolerance test (GTT) in the home to address transportation and childcare issues. In a similar vein, practitioners proposed a new category of outreach workers to act as GDM navigators and contribute to continuity of care. One provider also suggested the creation of a regional or institutional registry for PPGT that would make results available to obstetricians, patients, and their PC providers as a remedy for the challenge of transferring information between institutions, when obstetrician and PC provider work in different locations.

\section{CONCLUSIONS}

Results from this study show that women who have recently delivered may take their babies for well child visits, but often find it difficult to prioritize or obtain preventive care for themselves after a complicated pregnancy. Clinicians recognize the increased risk of T2DM onset following GDM, ${ }^{28}$ and the increased risk of hypertension following gestational hypertension or toxemia, ${ }^{29}$ but present systems of care do not incorporate strategies to bridge the gap between complicated obstetrical care and continued monitoring in a PC setting. ${ }^{30}$ Prevention of early onset has the potential for medical cost savings and improvements in quality of life.

Simply publishing guidelines for postpartum screening of T2DM is not enough. ${ }^{31}$ Research has shown the importance of women attending the postpartum visit in improving woman's chances for postpartum screening for diabetes, ${ }^{32} 3319$ but this visit may not take place. The concept of attention to women's health during the interconception/internatal period has gained ground, ${ }^{34}$ but interconception care only applies to women who may have a subsequent pregnancy, and is still prevention for the sake of a healthy pregnancy rather than the longterm preventive approach across the life cycle that women need to reduce their excess burden of chronic illness and disability in older age. Essentially, women's 
health, as Clancy wrote 23 years ago, ${ }^{35}$ is still a 'patchwork quilt with gaps.' Therefore, continuity of care and access to continuing monitoring for women after a diagnosis of GDM must be more robust than the current standard of care.

The recommendation to improve discharge instructions may be an important first step, since it requires no additional financial expense. Discharge is a crucial step along the way to continued care, and odds of being tested are associated with having GDM coded at hospital discharge. ${ }^{19}$ Studies also show that automated orders for postpartum testing on discharge, notification to providers, and telephone and email reminder messages may improve rates of postpartum testing, ${ }^{19} 33$ along with the creation of clinical protocols for postpartum testing. ${ }^{34}$

Many institutions have defaulted to HbA1C testing instead of the GTT in an effort to increase compliance with PPGT, but sensitivity of $22.64 \%$ and a positive predictive value of $54.55 \%$ against the GTT A1C test suggest that it does not provide a sensitive and specific diagnosis of abnormal carbohydrate metabolism in women who have had GDM. ${ }^{36}$ Furthermore, the very low rates of any testing that we found in our own institution among women at high risk of early onset indicate that a change in the guidelines for postpartum testing will not in itself be sufficient to effect change in transition to continued monitoring. A more effective change in guidelines might be the development of a reimbursement structure for an additional postpartum visit at 10-12 weeks for women with GDM; this additional visit might allow the obstetric clinician to focus beyond the immediate concerns after delivery to discuss prevention, set up an appointment for the GTT, and make an active referral to PC.

The proposal to move the postpartum test from laboratory to home merits exploration. This strategy supports the finding that simply reminding women to have a postpartum glucose test may not be enough. ${ }^{37}$ While there are no studies of the effectiveness of a visiting nurse administering the GTT, home visiting has already shown some success for women with pregnancy-induced hypertension, ${ }^{38}$ and diabetes educators are a recommended strategy for home management of diabetes for women diagnosed with GDM. ${ }^{39}$ Home visits may not be feasible, however, for women who return to work immediately. Telephone interventions focusing on diet, physical activity, and weight goals by coaches trained in physical activity, social cognitive strategies, and motivational interviewing techniques are currently under investigation, as well. ${ }^{40}$

One provider suggested a registry for consolidation of data, to allow transfer of test results from obstetrician to PC practitioner when each is housed in a different institution. Such a registry does not exist in the USA, but other countries have potential models for one, such as the Saudi National Diabetes Registry (SNDR), which not only acts as an epidemiological reporting system but also assesses the quality of healthcare for healthcare systems (report rates of diabetes control in patient population for particular healthcare institutions), economic impact of diabetes, and geographic distribution of cases. ${ }^{41}$

Finally, there is strong evidence from the results of this study and others of a need for widespread changes to electronic systems. First, there must be an effort toward data system linkages, ${ }^{4243}$ as previous research shows that simply estimating the prevalence of GDM is difficult, because the diagnosis can show up in birth certificate data, Medicaid data, or prenatal and discharge data in state health plans. ${ }^{44}$ Poor communication has already been reported among obstetricians and PCPs, ${ }^{45}$ suggesting a need for cross-communication among electronic health records, a barrier which is confirmed by the findings of this study. However, simply having crosscommunication is not enough. In a study by Stuebe et $a l,{ }^{45}$ only $45.8 \%$ of women with GDM as diagnosed by a GTT had that history documented on an EMR problem list, suggesting the need for improved EMR templates, supported by these results, and raising awareness among providers about the importance of documenting GDM histories.

In summary, patients and providers described major challenges to PPGT and transition to PC for continued monitoring. Key barriers included patients' low motivation for self-care and competing priorities. For obstetrical providers, the main concerns that emerged were the need to balance risk with reassurance and the seemingly irresolvable system issues related to the timing of the test, failures of documentation, and the lack of communication between obstetric and PC providers. Clinicians suggested introducing EMR templates for a GDM tailored 'meaningful use' postpartum discharge instructions, home-based testing, a GDM registry for tracking results, and the use of GDM navigators not currently available in the healthcare delivery system, which could all contribute to increasing PPGT rates and enhancing the likelihood of successful transition to PC for continued monitoring.

Acknowledgements The authors gratefully acknowledge the contributions of Project Managers Meryl St. John and Judy Margo, and Research Assistants Vicky Baudin, Myrdell Belizaire, Blaine Ferrari, Lynn Ibekwe, Marie Leinberger, Angelica Ramirez, Asari Offiong, Marlena Sherman, and Hannah.

Contributors JAB and LMC designed the study, collected and analyzed data, and wrote the manuscript. CMG contributed to the discussion section, and reviewed and edited the manuscript. REI and AL-P contributed to design and analysis, and reviewed/edited the manuscript.

Funding This work was supported in part by NICHD R21-HD75640. The study sponsor had no role in study design; collection, analysis, and interpretation of data; writing the report; or the decision to submit the report for publication.

Competing interests None declared.

Ethics approval Boston University Institutional Review Board.

Provenance and peer review Not commissioned; externally peer reviewed.

Data sharing statement Data entered into Dedoose software may be shared for research purposes only. Digital tapes have been destroyed to protect confidentiality. 
Open Access This is an Open Access article distributed in accordance with the Creative Commons Attribution Non Commercial (CC BY-NC 4.0) license, which permits others to distribute, remix, adapt, build upon this work noncommercially, and license their derivative works on different terms, provided the original work is properly cited and the use is non-commercial. See: http:// creativecommons.org/licenses/by-nc/4.0/

\section{REFERENCES}

1. Centers for Disease Control and Prevention. National diabetes fact sheet: National estimates and general information on diabetes and pre-diabetes in the United States, 2011. Atlanta, GA: U.S Department of Health and Human Services, Centers for Disease Control and Prevention, 2011.

2. Centers for Disease Control and Prevention. Diabetes and women's health across the life stages: a public health perspective. Atlanta, GA: U.S. Department of Health and Human Services, Centers for Disease Control and Prevention, 2001.

3. Cheung NW, Byth K. Population health significance of gestational diabetes. Diabetes Care 2003;26:2005-9.

4. American College of Obstetricians and Gynecologists Committee on Practice Bulletins-Obstetrics. ACOG Practice Bulletin. Clinical management guidelines for Obstetrician-Gynecologists. Number 30, September 2001 (replaces Technical Bulletin Number 200, December 1994): Gestational Diabetes. Obstet Gynecol 2001;98:525-38.

5. Leddy MA, Power ML, Schulkin J. The impact of maternal obesity on maternal and fetal health. Rev Obstet Gynecol 2008;1:170-8

6. Kim C, Newton KM, Knopp RH. Gestational diabetes and incidence of type 2 diabetes mellitus: a systematic review. Diabetes Care 2002;25:1862-8.

7. Bellamy L, Casas JP, Hingorani AD, et al. Type 2 diabetes mellitus after gestational diabetes: a systematic review and meta-analysis. Lancet 2009;373:1773-9.

8. Kim C, Berger DK, Chamany S. Recurrence of gestational diabetes mellitus: a systematic review. Diabetes Care 2007;30:1314-19.

9. Getahun D, Fassett MJ, Jacobsen SJ. Gestational diabetes: risk of recurrence in subsequent pregnancies. Am J Obstet Gynecol 2010;203:467.e1-6.

10. Kim C, Herman WH, Vijan S. Efficacy and cost of postpartum screening strategies for diabetes among women with histories of gestational diabetes mellitus. Diabetes Care 2007;30:1102-6.

11. Golden SH, Bennett WL, Baptist-Roberts K, et al. Antepartum glucose tolerance test results as predictors of type 2 diabetes mellitus in women with a history of gestational diabetes mellitus: a systematic review. Gender Med 2009;6:109-22.

12. Ferrara A, Kahn HS, Quesenberry CP, et al. An increase in the incidence of gestational diabetes melitus: Northern California, 1991-2000. Obstet Gynecol 2004;103:526-33.

13. Xiang $\mathrm{AH}$, $\mathrm{Li} \mathrm{BH}$, Black $\mathrm{MH}$, et al. Racial and ethnic disparities in diabetes risk after gestational diabetes mellitus. Diabetologia 2011;54:3016-21.

14. Ratner RE, Christophi CA, Metzger BE, et al. Prevention of diabetes in women with a history of gestational diabetes: effects of metformin and lifestyle interventions. J Clin Endocrinol Metab 2008;93:4774-9.

15. Anna V, van der Ploegh HP, Cheung NW, et al. Sociodemographic correlates of the increasing trend in prevalence of gestational diabetes mellitus in a large population of women between 1995 and 2005. Diabetes Care 2008;31:2288-93.

16. Dacus JV, Meyer NL, Muram D, et al. Gestational diabetes: postpartum glucose tolerance testing. Am J Obstet Gynecol 1994:171:927-31.

17. Kjos SL, Buchanan TA, Greenspoot JS, et al. Gestational diabetes mellitus: the prevalence of glucose intolerance in the first two months postpartum. Am J Obstet Gynecol 1990;63:93-8.

18. Hunt KJ, Conway DL. Who returns for postpartum glucose screening following gestational diabetes mellitus? Am J Obstet Gynecol 2008;198:404.e1-e6.

19. Lawrence JM, Black MH, Hsu JW, et al. Prevalence and timing of postpartum glucose testing and sustained glucose dysregulation after gestational diabetes mellitus. Diabetes Care 2010;33:569-76.

20. Ferrara A, Peng T, Kim C. Trends in postpartum diabetes screening and subsequent diabetes and impaired fasting glucose among women with histories of gestational diabetes mellitus: a report from the Translating Research Into Action for Diabetes (TRIAD) Study. Diabetes Care 2009;32:269-74.

21. American Diabetes Association. Gestational diabetes mellitus. Diabetes Care 2004;27:S88-90.
22. Shah BR, Lipscombe LL, Feig DS, et al. Missed opportunities for type 2 diabetes testing following gestational diabetes: a population-based cohort study. BJOG 2011;118:1484-90.

23. Tovar A, Chasan-Taber L, Eggleston E, et al. Postpartum screening for diabetes among women with a history of gestational diabetes mellitus. Prev Chronic Dis 2011;8:A124.

24. McCloskey L, Bernstein J, Winter M, et al. Follow-up of gestational diabetes mellitus in an urban safety net hospital: missed opportunities to launch preventive care for women. J Womens Health (Larchmt) 2014;23:327-34.

25. Gillman MW, Rifas-Shiman S, Berkey CS, et al. Maternal gestational diabetes, birth weight, and adolescent obesity. Pediatrics 2003;111: e221-6.

26. Damschroder LJ, Aron DC, Keith RE, et al. Fostering implementation of health services research findings into practice: a consolidated framework for advancing implementation science. Implement Sci 2009;4:50-65.

27. Patton MQ. Qualitative evaluation and research methods. 2nd edn. Thousand Oaks, CA: Sage, 1990.

28. Feig DS, Zinman B, Wang X, et al. Risk of development of diabetes mellitus after diagnosis of gestational diabetes. CMAJ 2008;179:229-34.

29. Van Oostwaard MF, Langenveld J, Schuit E, et al. Prediction of recurrence of hypertensive disorders of pregnancy in the term period, a retrospective cohort study. Pregnancy Hypertens 2014:4:194-202.

30. Ehrenthal DB, Maiden K, Rogers S, et al. Postpartum healthcare after gestational diabetes and hypertension. $J$ Womens Health (Larchmt) 2014;9:760-4.

31. McGovern A, Butler L, Jones S, et al. Diabetes screening after gestational diabetes in England: a quantitative retrospective cohort study. Br J Gen Pract 2014;64:e17-23.

32. Hale NL, Probst JC, Liu J, et al. Postpartum screening for diabetes among Medicaid-eligible South Carolina women with gestational diabetes. Womens Health Issues 2012;22:e163-9.

33. Ko JY, Dietz PM, Conrey EJ, et al. Strategies associated with higher postpartum glucose tolerance screening rates for gestational diabetes mellitus patients. $J$ Womens Health (Larchmt) 2013;22:681-6.

34. Lu MC, Kotelchuck M, Culhane JF, et al. Preconception care between pregnancies: the content of internatal care. Matern Child Health J 2006;10:S107-22.

35. Clancy CM, Massion CT. American women's health care. A patchwork quilt with gaps. JAMA 1992;268:1918-20.

36. Picón MJ, Murri M, Muñnoz A, et al. Hemoglobin A1c versus oral glucose tolerance test in postpartum diabetes screening. Diabetes Care 2012;35:1648-53

37. Van Ryswyk EM, Middleton PF, Hague WM, et al. Postpartum SMS reminders to women who have experienced gestational diabetes to test for type 2 diabetes: the DIAMIND randomized trial. Diabet Med 2015;32:1368-76.

38. Kitzman $\mathrm{H}$, Olds DL, Henderson $\mathrm{CR}$, et al. Effect of prenatal and infancy home visitation by nurses on pregnancy outcomes, childhood injuries, and repeated childbearing. A randomized controlled trial. JAMA 1997;278:644-52.

39. Armstrong CL, Brown LP, York R, et al. From diagnosis to home management: nutritional considerations for women with gestational diabetes. Diabetes Educ 1991;17:455-9.

40. Ferrara $\mathrm{A}$, Hedderson $\mathrm{MM}$, Albright $\mathrm{CL}$, et al. A pragmatic cluster randomized clinical trial of diabetes prevention strategies for women with gestational diabetes: design and rationale of the Gestational Diabetes' Effects on Moms (GEM) study. BMC Pregnancy Childbirth 2014;14:21.

41. Al-Rubeaan KA, Youssef AM, Subhani SN, et al. A web-based interactive diabetes registry for health care management and planning in Saudi Arabia. J Med Internet Res 2013;15:e202.

42. Devlin HM, Desai J, Walaszek A. Reviewing performance of birth certificate and hospital discharge data to identify births complicated by maternal diabetes. Matern Child Health $J$ 2009;13:660-6.

43. Lydon-Rochelle MT, Holt VL, Cárdenas V, et al. The reporting of pre-existing maternal medical conditions and complications of pregnancy on birth certificates and in hospital discharge data. Am J Obstet Gynecol 2005;193:125-34.

44. Lynch CP, Baker N, Korte JE, et al. Increasing prevalence of diabetes during pregnancy in South Carolina. $J$ Womens Health (Larchmt) 2015;24:316-23.

45. Stuebe A, Ecker J, Bates DW, et al. Barriers to follow-up for women with a history of gestational diabetes. Am J Perinatol 2010;27:705-10. 